THE ELECTRO-MAGNETIC TELEPHONE TRANSMITTER.

AN interesting contribution was made by M. Merca-
dier in a recent number of the Comptes Rendus de l'Academie Francaise. On the ground of some nove and some already accepted experimental eveidence, $M$ Mercadier holds that the mechanism by virtue of ments is analogous to, if not identical with, that by which solid bodies of any form, a wall for instance,
transmit to one of their surfaces all the vibratory movements of any kind which are produced in the ai in contact with the other surface. It is a phenomenon
or resonance. Movements corresponding to particular sounds may be superposed in slender diaphragms, but this superposition must necessarily be disturbing unthis view, it is cited that diaphragms much too rigid, or charged with irregularly distributed masses over the surface, or pierced with holes, or otherwise evidently unThey will likewise serve when feathers, wool, wood, metals, mica, and other substances to the diaphragm and the source of vibratory movement. The magnetic fleld does not alter these be removed altogether. It is sufficient to replace it by with a piece of pasteboard or paper. Such a telephone works distinetly although feebly; but any slender flexing of the cover of the instrument, with one or two yield results of increased and even ordinary inten will This is the iron filing telephone, which is reversible; for a given magnetic field there is a certain weight of that the advantage of the iron diaphragm over iron
filings reduces itself to presenting in a certain volume
a much more considerable number of magnetic molecules to the action of the field. The iron diaphragm increases the telephonic intensity, but it is by no means

\section{ON ELECTRO-DISSOLUTION, AND ITS USE AS REGARDS ANALYSIS.}

By H. N. WARREN, Research Analyst.

ON the same principle that electro-dissolution is used have lately varied the experiment by introducing, of boron, and, having connected the respective boride with the positive pole of a powerful battery, and to
the negative a plate of platinum, using as a solvent dilute sulphuric acid, I observed, after the lapse of solution, and a considerable amount of brownish precipitate had collected at the bottom of the vessel, intercepted by flakes of graphite and carbon; the
precipitate, having been collected on a filter paper, washed, and dried, on examination proved to be amorphous boron, containing graphite and other impurithe preparation of the boron compound. The boron intensely heated in a current of hydrogen gas, for the purpose of rendering it more dense and destroying into a combustion tubing, heated to bright redness, in order to separate the carbon, finally pure boron In like manner silicon-eisen, containing 9 per cent. of silicon, was treated, but not giving so satisfactory a
result. A small quantity only of silicon separates in the uncombined form, the greater quantity separating
in the form of silica, $\mathrm{SiO}_{2}$, the amorphous silicon so obtained apparently being more prone to oxidation than the boron so obtained.
Ferrous sulphide was next similarly treated, and gave, after the lapse of a few hours, a copious blackish precipitation of sulphur, and possessing properties
similar to the sulphur obtained by dissolving sulphides such as cupric sulphide in dilute nitric acid, in all othe
respects resembling common sulphur. Phosphides of iron zine sto wer.

and gave, besides earbon and other impuritice, residue containing a large percentage of phosphorus residue containing a large percentage of phosphorus,
which differed from ordinary phosphorus with respect
to its insolubility in carbon disulphide, and which resembled the reaction in the case with silicon-eisen rather than that of the boron compound, insomuch
that a large quantity of the phosphorus had passed into solution. hydrochloric acid as a solvent in place of sulphuric acid. In the course of a day the copper had entirely dissolved and precipitated itself on the negative copper, after having been washed, dried, and weighed, gave identical results with regard to percentage with
a careful gravimetric estimation. I have lately used respect to the analysis of commercial copper, especially in the estimation of small quantities of arsenic, thus enabling the experimenter to perform his investigais resorted to, at the same time avoiding the precipitated copper carrying down with it thearsenic. I have in this manner detected arsenic in commercial copper when all other mothods have totally failed. I have
also found the above method especially applicable with respect to the analysis of brass. will briefly mention, a rod composed of an alloy of becoming entirely dissolved and precipitating itself on the platinum electrode, the whole of the silver remain ing suspended to the positive electrode in an abores
cent form. Arsenide of zine was similarly treated, th platinum electrode. Various otheralloys, being experi-

I may also, in the last instance, mention that $I$ hav

lound the above methods of electro-dissolution pecu- \begin{tabular}{|l|l} 
liarly adapted for the preparation of unstable com- & influence on the quantity of the secretion passing away \\
pounds such as stannic nitrate, potassic ferrate, ferric & An increased rate of moveunent on the part of the blood
\end{tabular} heat, and in some instances have succeeded by the ty of urine. On a solution of common salt or of mere following means of crystallizing the resulting com-
pound obtuined.-Chem. News.

A NEWLY DISCOVERED SUBSTANCE IN URINE.

DR. LEO's researches on sugar in urine are interestng, and tend to correct the commonly accepted views
the subject. Professor Scheibler, a chemist well the determination of the quantity of that substance contained in a liquid gives different results, according as it is done by Trommers method or with the polar
scope. As sugar nowadays is exclusively dealt with ac cording to the degree of polarizatiom, this fact is of tance that is more powerful in that haspect than grape sugar. Dr. Leo's researches yield analogous results, uantity of diabetic urine after three different methods namely, Trommer's (alkaline solution of copper); by many cases the results agreed, while in others there was He succeeded in isolating a substance correspondin
He $n$ its chemical composition to grape sugar, and also a and turning the plane of polarization to the left. The power of reduction of this newly discovered substance
is to that of grape sugar as $1: 2448$. Dr. Leo found this s to that of grape sugar as $1: 2 \cdot 48$. Dr. Leo found this
substance in three specimens of diabetic urine, but it was absent in normal urine, although a great amount as examined for that purpose. From this it may be the organism, and that it is a pathological product
The theory of Dr. Jaques Meyer, of Carlsbad, that it may be connected with obesity, is negatived by th was found, only one was corpulent

\section{FURNACE FOR DECOMPOSING CHLORIDE} OF MAGNESIUM

THE problem of decomposing chloride of magnesium is one which has attracted the attention of technical
chemists for many years. The solution of this problem ould be of great importance to the alkali trade, and, Weldon made many experiments on this subject, but

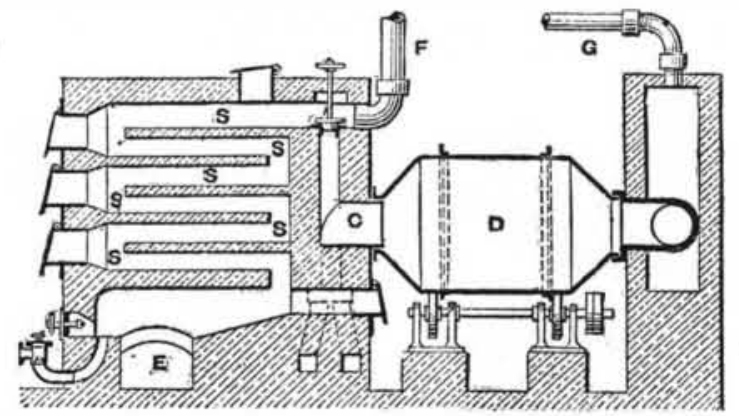

without any particular success. Of late a furnace has been patented in Germany, by A. Vogt, which is
worked on a principle similar to that applied to salt
cake furnaces; but with this difference that in place of the pot it has a revolving drum, and instead of th roaster a furnace with a number of shelves. The heating gases are furnished by a producer, and pass from centrated chloride of magnesium. When the latter has solidified, but before being to any extent decomposed, shelf of the furnace. It is then gradually removed one shelf lower as the decomposition increases, until it
arrives at the bottom shelf, where it is completely de composed in the state of magnesia, which is emptied
through, $\mathrm{E}$. The drum, $\mathrm{D}$, after being emptied, is again filled with concentrated solution of chloride of magneIf, instead of hydrochloric acid, chlorine is to b evolved, it is necessary to heat the furnace by means of
hot air, as otherwise the carbonic acid in the gases from the generator would prevent the formation of
bleaching powder. The air is heated in two regenerating chamber
Industries.

THE FILTRATION AND THE SECRETION THEORY.

AT a recent meeting of the Physiological Society, Dr J. Munk reported on experiments instituted by him in at an experimental decision between the two theories of the secretion of urine-the filtration theory of Lud-
wig and the secretion theory of Heidenhain. Accordwig and the secretion theory of Heidenhain. Accord-
ing to the first theory, the blood pressure prescribed
the measure for the urine secretion; according to the the measure for the urine secretion; according to the epithelial cells of the kidneys, and the quantity of the matter secreted was dependent on the rate of moveinstituted his experiments on excided but living kidof the same animals, under pressures which he was able
to vary at pleasure between $80 \mathrm{~mm}$. and $190 \mathrm{~mm}$. Fifty experiments on dogs whose blood and kidneys were,
during the experiment, kept at $40^{\circ}$ C., yielded the reduring the experiment, kept at $40^{\circ}$ C., yielded the reitself in copious quantities where normal blood was conducted through the kidney. If to the famished
blood was added one of the substances contained a blood was added one of the substances contained as
ultimate products of digestion in the blood, such, for example, as urea, then did the secretion ensue.
The fluid dropping from the ureter contained mor The fluid dropping from the ureter contained more
urea than did the blood. That fluid was therefore ro urea than did the blood. That fluid was therefore no that the molecules of each substance enter into the
filtrate, but a secretion. An enhancement of the pres-
crystal retaining all the optical properties which ercised from the heart or from the nervous systern on the kidneys, were deemed by the speaker arguments pial cells. A series of diuretics was next tried, in order to establish whether they operated in the way of stimulus centrally on the heart or peripherally on the renal
cells. Digitalis was a central diuretic. Common salt, on the other hand, was a peripheral diuretic. Added the quantity of urine eight to fifteen fold. Even in much less doses, it was a powerful diuretic. In a similar manner, if yet not so intensely, operated saltpeter and duction, however, of the last substance into the blood measure as was the quantity of urine increased, so that in this case the increase in the quantity of urine was, perhaps, exclusively conditioned by the greater speed
in the movement of the blood. On the otherhand, the quantity of secreted urine was reduced when morphine of the application of strychnine, the rate in the current reduction in the secretion of the urine.
The speaker had, finally, demonstrated the synthesis of hippuric acid and sulphate of phenol in the excided kidney as a function of its cells, by adding to the blood pouring through the kidney, in the first place, benzoic phate of soda. In order that these syntheses might presence of the blood corpuscles was not necessary,
though, indeed, the presence of oxygen in the blood though, indeed, the

VARYING CYLINDRICAL LENS.

By Tempest Anderson, M.D., B.Sc.

THE author has had constructed a cylindrical lens in which the axis remains constant in direction and ridian at right angles to this varies continuously. A cone may be regarded as a succession of cylinders
of different diameters graduating into one another by exceedingly small steps, so that if a short enough portion be considered, its curvature at any point may be and the other ground on a conical tool is therefore concave cylindrical lens varying in concavity at differ-
ent parts according to the diameter of the cone at the corresponding part. Two such lenses mounted with axes parallel and with curvatures varying in opposite refraction in the direction of the axes is zero, and whose refraction in the meridian at right angles to this is at any point the sum of the refractions of the two lenses. This sum is nearly constant for a considerable distance is maintained. If the lenses be slid one over the other in the direction of their axes, this sum changes, and we have a varying eylindrical lens, The lens is graduated tralized.

Lenses were exhibited to the Royal Society, London,
varying from 0 to $-6 \mathrm{DCy}$, and from 0 to +6 DCy.

THE LAWS OF THE ABSORPTION OF LIGHT IN CRYSTALS. By H. BecQuerel.

1. THE absorption spectrum observed through a minous vibration which propagates itself in this cryscrystal have, in the spectrum, fixed positions, their
intensity alone varying. 3. For a given band or ray there exist in the crystal three rectangular directions of symmetry, according to one of which the band gene-
rally disappears, so that for a suitable direction of the radiations corresponding to the no longer absorbs the where the band question appeared. These three direc tions may be called the principal directions of absorpcrystals, by a this bavd. 4. In the orthorhombic symmetry, the principal directions of absorption of all the bands coincide with the three axes of symmetry.
We may thus observe three principal absorption spectra. In uniaxial crystals the number of absorption specof the principal directions of absorption of each crystal coincides with the only axis of symmetry; the two may be found variously disposed in the plane normal
to this axis. Most commonly these principal direction are very near to the principal corresponding directions of optical elasticity. 6. In various crystals the charac-
ters of the absorption phenomena differ strikingly from those which we might expect to find after an examination of the optical properties of the crystal. We have orption directions of certain bands were completely different from the axis of optical elasticity of the crysthis anomaly, we perceive that the crystals manifesting these effects are complex bodies, formed of various
matters, one, or sometimes several, of which absorb light and give each different absorption bands. Now, morphism of certain substances does not necessarily involve identity of optical properties, and in particuar in the directions of the axes of optical elasticity in a crystal containing a mixture of isomorphous substances, each substance brings its own influence, which
may be made to predominate in turn according to the may be made to predominate in turn according to the
proportions of the mixture. We may, therefore, admit 